\title{
El objeto de estudio como sustento esencial de la investigación en Comunicación
}

\author{
The object of study as an essential support \\ Communication Research
}

\author{
Dr. José Antonio Meyer Rodríguez \\ Universidad Popular Autónoma del Estado de Puebla (México) \\ joseantonio.meyer@gmail.com
}

Recibido: 10 de Noviembre de 2010

Aceptado: 30 de Noviembre de 2010

\section{Resumen}

Este artículo expone un método para la construcción consistente y sustentable de objetos de estudio en el área de Comunicación. El esquema ratifica la necesidad de delimitar el objeto de interés a partir de preguntas que aseguren la rigurosidad inherente a cualquier proceso de investigación. En este subyacen los aspectos epistemológicos, teóricos, metodológicos y empíricos, así como una postura neutral ante los debates metodológicos.

\section{Abstract}

This paper presents a method for build consistent and sustainable objects to study in the communication area. The diagram confirms the necessary delimitation at the object based on questions to ensure the inherent rigor of any research. In it underlies the epistemological, theoretical, methodological and empirical own research, while define a neutral position about the methodological debates.

\section{Key Words}

Objects to study, Analysis methods, Knowledge construction, Research questions, Methodological design.

\section{Palabras clave}


Objeto de estudio, método de análisis, construcción de conocimientos, preguntas de investigación, diseño metodológico.

\section{Planteamiento general}

Aspecto de especial significación en la investigación de los procesos y fenómenos comunicativos, es lo que muchos especialistas han definido como la delimitación de objetos de estudio (Domínguez, 2007, Hernández y Barriga, 2003, Díaz, 1997, Bourdieu, Chamboredon y Passeron 1996 e Hidalgo, 1992). Esta construcción, que va más allá de lo metodológico, constituye una búsqueda constante por parte del investigador para alcanzar la mayor precisión, claridad y orientación en sus trabajos de valor científico. El asunto es de la mayor importancia para esta área de estudio, toda vez que todavía se encuentra una escasez significativa en las delimitaciones epistemológicas, precarias discusiones teóricas, poca sustentación experimental y, sobre todo, limitados esfuerzos porque los análisis constituyan verdaderas aportaciones para las líneas de investigación existente y el avance de la disciplina. En esa dimensión, este artículo define algunas orientaciones necesarias para la construcción consistente y sustentada de objetos de estudio en el área de Comunicación.

\subsection{Primera orientación}

En principio es necesario señalar que si bien es importante lo qué se investiga, también es relevante la manera cómo se investigan y definen los objetos de estudio para que sean realmente significativos y favorezcan el avance de la disciplina. Ello tiene que ver, como lo refiere (Lozano, 1996), con el procesamiento analítico del mismo, pero también con la estructuración de un esquema de discusión y problematización que confronte y provoque al investigador a realizar análisis con un mayor nivel de profundidad. Esta forma de trabajo reconoce la pertinencia de un proceso de construcción de conocimientos que otorgue mayor valor al cómo del proceso y su consiguiente organización desde la perspectiva de un investigador activo. En tal forma, delimitar objetos de estudio no es repetir mecánicamente algunos pasos de carácter metodológico sino abordar un problema o fenómeno a partir de textos teóricos referentes con el propósito de contribuir al avance de una línea de investigación en esta área del conocimiento. Es importante considerar, como lo dicen Bourdieu, Chamboredon y Passeron (1996:p.26), que la investigación es un constructo intelectual, es decir, "un proceso constante de construcción, reflexión y, en muchos casos, de 
improvisación ante lo imprevisto". Por ello, vale la pena reiterar nuevamente lo primordial que resulta el método en cualquier construcción humana y que la investigación constituye un proceso de tipo artesanal, no un conjunto estricto de pasos determinados.

Hernández y Barriga (2003:p6) han definido que "el método es una forma de trabajo para abordar el objeto de estudio mediante preguntas que aseguren la rigurosidad inherente a cualquier proceso de investigación". Esta distinción no establece modalidades mutuamente excluyentes, sino verdaderas orientaciones de fondo que son sumamente importantes al momento de establecer las herramientas necesarias para el desarrollo de una investigación significativa. Esta especie de oficio artesanal requerido para enfrentar imprevistos resulta esencial al momento de que los objetivos no son fácilmente alcanzados con modelos previamente establecidos. Por ello, Sánchez Puentes (2004:p.58) señala que el desarrollo de una perspectiva activa frente a la investigación actúa siempre como un eje orientador en el uso de distintas metodologías. El surgimiento de un objeto de estudio es siempre el resultado de una inquietud epistemológica más que metodológica. Esto quiere decir que "representar el objeto de estudio implica necesariamente identificar la conjunción de fuerzas que conlleva realizar una investigación científica". Una de las primeras consideraciones al respecto consiste en identificar las diferencias concretas entre lo que Bourdieu, Chamboredon y Passeron (1996) han denominado el objeto artesanal y el objeto prefabricado. Estas reflejan un referente esencial entre el quehacer de la investigación y su relación con el origen de las inquietudes que la impulsan, sean estas propias o ajenas. El objeto artesanal nace de intereses propios, cualquiera que sea su origen, el prefabricado, en cambio, surge de los intereses de otros actores sociales -como los organismos gubernamentales, los grupos sociales o de investigación- cuyo estudio es solicitado en algunas ocasiones a los especialistas universitarios. Sierra Bravo (2007) manifiesta que es importante superar la forma tradicional de argumentar sobre el método científico para asumir una visión más abierta y pluralista que enfatice menos en la actividad y más en las preguntas de investigación. Esta concepción se centra en las cuatro interrogantes que todo investigador en Comunicación ha de considerar al momento de leer sobre estudios realizados o en el proceso de formular una nueva investigación: 


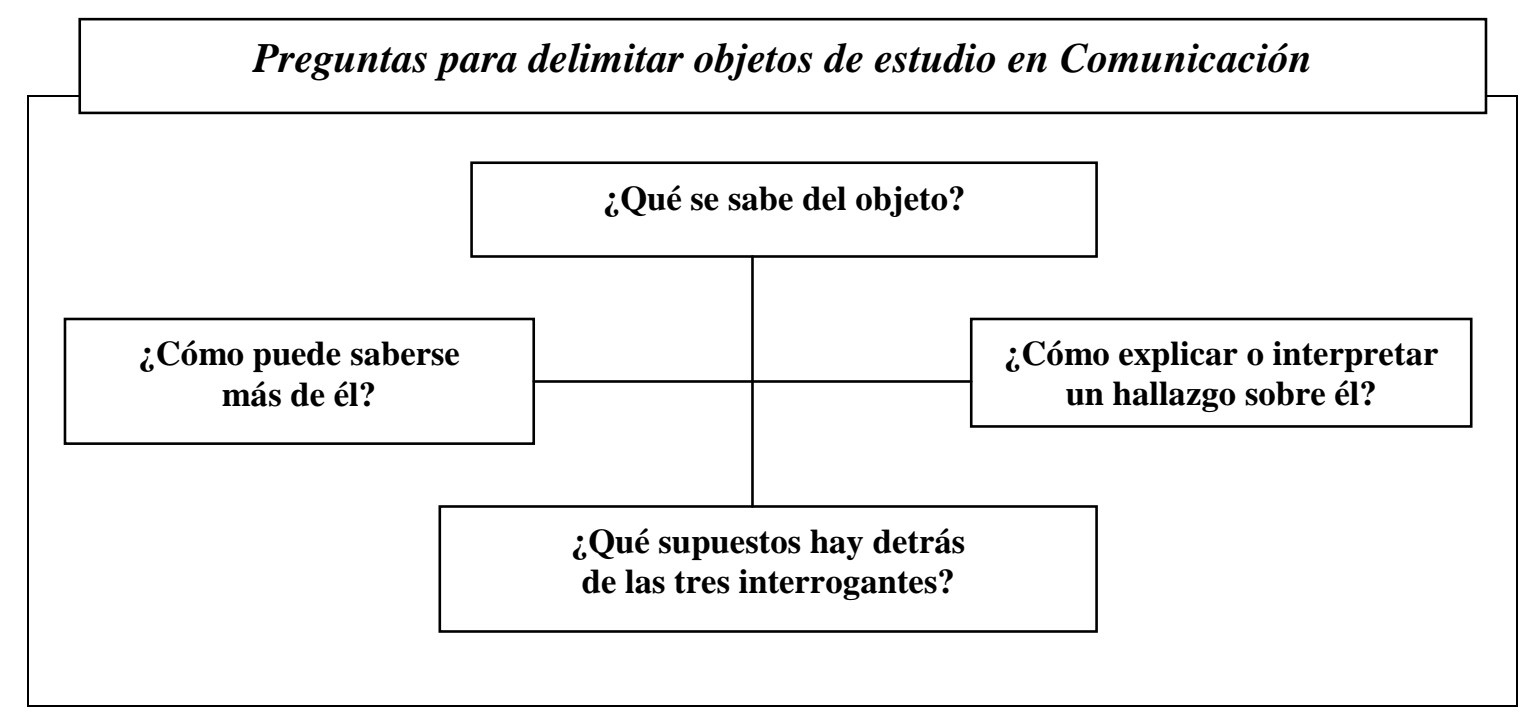

En esas preguntas subyacen los aspectos epistemológicos, teóricos, metodológicos y empíricos propios de una investigación, pero también una postura neutra ante los debates cuantitativos y cualitativos. Por tanto, Hernández y Barriga (2003) destacan la congruencia lógica de un estudio que es fundamental al momento de definir la forma más adecuada para realizarla.

Delimitación de un objeto de estudio en Comunicación

Elementos epistemológicos

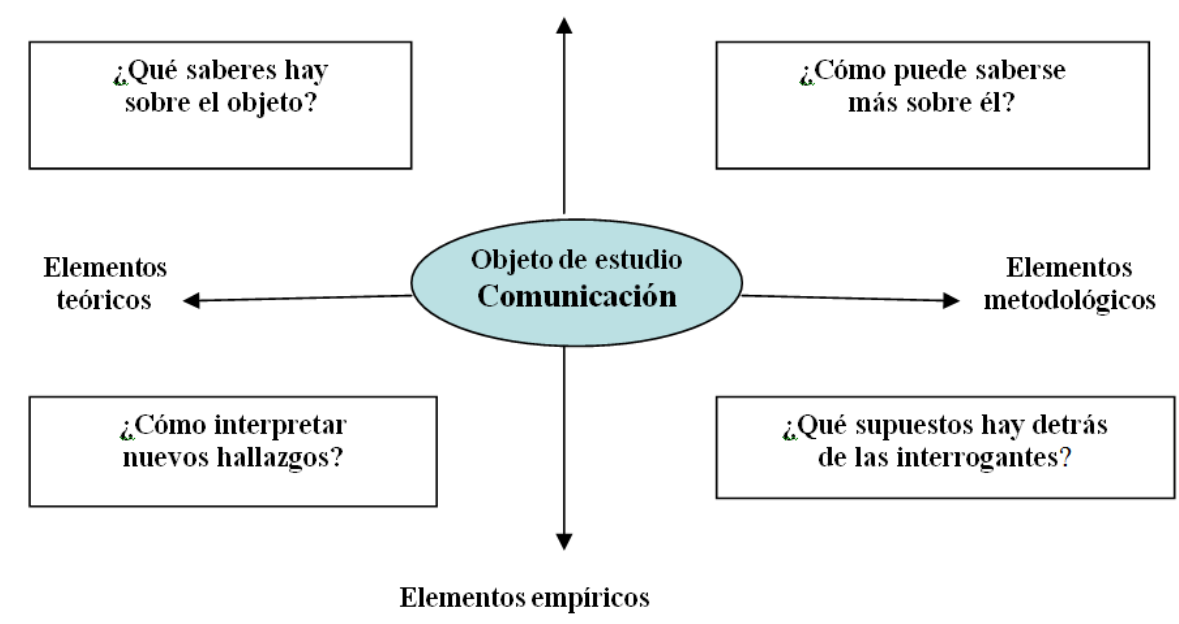

JAMR/2010 
Al plantearse estas preguntas como núcleo básico de la investigación, el analista puede decidir lo que desea conocer. De esta manera el quehacer científico se desmitifica y convierte, no sólo en una actividad que cumple con ciertos parámetros previamente definidos, sino en un desarrollo conceptual e instrumental que puede ser evaluado por cualquier lector informado en función de la coherencia del trabajo y la concatenación de los cuatro ejes centrales de la ciencia. Asimismo, como lo expresa Hidalgo (1992), las respuestas a las cuatro interrogantes puede indicar con transparencia la forma como el investigador construyó su objeto de estudio en razón de cierto tipo de intereses claramente expuestos y manifiestos. Ante ello, es posible precisar que un objeto de estudio es siempre el componente de un fenómeno/problema/proceso comunicacional sobre el que desea saberse o conocerse. Esta formulación, en cierto medida obvia, es también la distinción entre lo que se conoce como un objeto artesanal y otro prefabricado. Hoyos (2005:p.15) manifiesta que desde una perspectiva constructivista "el conocimiento parte del supuesto que la investigación es una forma de moldear la representación de un fenómeno de interés. En consonancia, la investigación científica busca enfatizar sobre la naturaleza transparente y criticable de la forma como se construye el objeto". Esto quiere decir que mientras el fenómeno de interés es el objeto de estudio y lo que se quiere saber, la forma de construir su representación es el proceso de investigación en sí mismo, con toda la complejidad empírica, metodológica, teórica y epistemológica que esto implica.

\subsection{Segunda observación}

Durante mucho tiempo el procedimiento científico tradicional se ha definido por una serie de componentes íntimamente relacionados que han terminado por esquematizar muchas de las investigaciones. En ese sentido, el reto consiste en transitar de esa extensión de conclusiones o generalización de resultados -fuertemente influenciado por el modelo hipotético-deductivo (Klimovsky, 1997 y Popper, 1993)- hacia un esquema de indagación artesanal más amplio y diverso que busque identificar los temas de interés y mayor relevancia significativa para el área de conocimiento. El revisar y discutir la bibliografía más actualizada y referente, desarrollar una visión sistémica y conceptual de lo que se sabe sobre el tema y entender cómo se llegó a saber sobre él, cómo se interpretó y qué supuestos hay detrás de esas respuestas, resulta esencial en esta orientación. 
En efecto, la revisión bibliográfica resulta sustancial si se centra en las cuatro dimensiones básicas de la ciencia y enfoca, como lo precisa De Alba (1996), en el cumplimiento de las siguientes funciones:

- Desarrollar una discusión teórica donde se contrasten posturas de diferentes autores

- Elaborar un marco referencial de cómo se piensa abordar el tema

- Delimitar el objeto de estudio a construir

- Definir objetivos generales y específicos, así como las hipótesis que permitan determinar el objeto conceptual

- Diseñar un esquema metodológico sobre el objeto empírico

- Recopilar la información necesaria

- Procesar la información y generar resultados

- Analizar los resultados y otorgarles sentido a partir de un marco referencial

- Redimensionar el objeto de estudio

- Elaborar y difundir el informe de investigación.

Aunque esta formulación no se ajusta a la visión positivista de lo que constituye "la ciencia", la hipótesis si se entiende -como lo define Navarro (2006:p.181)- en "un conjunto de ideas orientadoras que ayudan a guiar la investigación”. En ese sentido, el objeto de estudio constituye lo que se quiere saber y el espacio simbólico de la realidad que quiere abordarse de una forma científica. Como tal, es el resultado final de un proceso de investigación que requiere ser elaborado con rigurosidad, congruencia y sustentación. Esto es, interpretado epistemológicamente, manufacturado en forma teórica y conceptual, delimitado metodológicamente y determinado en forma empírica. En cada una de esas cuatro versiones del objeto es posible alcanzar de forma explícita o implícita lo siguiente:

- Definición del caso o casos que desean observarse

- Definición de las características que quieren observarse de esos casos

- Definición de lo que quiere hacerse con esas observaciones

- Definición del contexto en el que quieren hacerse esas observaciones. 
Galindo (2009) dice que la mejor forma de aproximarse a un objeto de estudio es mediante la formulación de preguntas. Así, por ejemplo, en el enunciado ¿qué relación existe entre los consumos intensivos de televisión de entretenimiento y el rendimiento escolar de los niños de educación primaria en las escuelas públicas de una ciudad determinada?, se destaca lo que desea saberse. A él se incorporan las nociones de casos (niños de educación primaria), las características a observar (consumos intensivos de televisión de entretenimiento), lo que quiere hacerse con las observaciones (establecer la relación entre el rendimiento escolar y la exposición al medio) y el contexto específico (escuelas públicas de una ciudad determinada). La pregunta reformulada como afirmación constituye el objeto de estudio a construir: "Relación entre el rendimiento escolar y los consumos intensivos de televisión de entretenimiento en los niños de educación primaria de escuelas públicas de una ciudad determinada". Es también un título adecuado para una investigación específica sobre el tema.

La delimitación del objeto supone también una inquietud por parte del investigador que lo lleva a definir una cierta temática de interés e invertir recursos para tratar de aprehenderla. No obstante, contrario a la práctica común, es propicio iniciar la búsqueda bibliográfica a partir de textos empíricos sobre el tema, de reciente autoría y publicados en revistas periódicas arbitradas. En ellas se consignan generalmente resultados de investigación sobre distintos temas y son, en gran medida, las fuentes de información más confiables. Ellas expresan los supuestos en que han sustentado sus aproximaciones y, sobre todo, las que identifican más precisamente los textos claves en la literatura existente. Dado ello, es posible plantear algunas estrategias que ayudan a identificar textos relevantes. Una de las más simples es la siguiente:

\begin{tabular}{|c|c|c|}
\hline \multirow{2}{*}{} & \multicolumn{2}{|c|}{ Fenómeno de interés } \\
\cline { 2 - 3 } & Mismo & Similar \\
\hline Mismo & Primera opción & Segunda opción \\
\hline Similar & Segunda opción & Tercera opción \\
\hline
\end{tabular}

De esta manera, una primera opción siempre es ubicar textos sobre el tema y contexto que interesa. Si eso no es posible, la opción es mantener el mismo fenómeno o contexto y buscar contextos o fenómenos similares. Otra opción es identificar literatura sobre fenómenos similares en contextos similares. Así, por ejemplo, si interesa estudiar el establecimiento de la agenda pública por parte de los principales noticieros de radio en una ciudad y su incidencia en la formulación de percepciones ciudadanas, lo mejor es encontrar textos sobre 
la construcción de la agenda pública en los medios de comunicación de esa ciudad. Si no se consiguen, pueden entonces localizarse textos sobre el tema en ciudades similares (mismo fenómeno y contexto similar) o medios de comunicación en esa ciudad (mismo contexto y fenómeno similar). En caso de no dar resultado, lo mejor es identificar textos sobre el tema en otros países. Esto es importante porque a menudo los aportes más interesantes provienen de fuentes que están aparentemente muy lejanas de la zona de interés primordial.

\subsection{Tercera observación}

Una vez identificados los textos que parecen ser los más útiles mediante una búsqueda intensa en bancos de datos o repositorios de revistas especializadas (ISI, SCOPUS, EBSCO, Dialnet, Redalyc u otros), el segundo paso es realizar las fichas bibliográficas. Aguilar (2007) sugiere que en ese proceso se respete el desglose conceptual de las cuatro grandes áreas de estudio (empírica, metodológica, teórica y epistemológica), cuyo desglose depende en gran medida del investigador y sus intereses. En esa medida, por ejemplo, el rubro de metodología puede subdividirse en operacionalización de variables, selección de casos, recolección de información, construcción y procesamiento de datos. La teórica puede agruparse en conceptos sustantivos, paradigmas teóricos, aspectos referenciales y puntos de debate e inflexión. Posterior a la clasificación de textos, el próximo paso consiste en la discusión bibliográfica, la cual contribuye a centrar aún más el objeto de estudio y encontrar la vertiente de mayor interés y significación. Al clasificar los textos y contrastar lo que diferentes autores dicen sobre los variados aspectos de un tema, no es de sorprender que ellos sean discordantes en sus posturas. La discusión no es solamente un ejercicio intelectual, sino algo sustancial que permite conocer diferentes posturas y, sobre todo, fundamentar una propia frente al tema de estudio. Karam (2008) destaca la importancia de decidir cuál de ellos puede ser el más acertado y por qué, en caso de que los hallazgos empíricos sean contradictorios. Siempre es conveniente pronunciarse en favor o en contra de una de ellas, con base en una argumentación sólida que elimine cualquier posición de tipo intituitivo. A veces el aporte consiste en vincular la temática con una teoría previamente contemplada, o incluso sintetizar posturas contradictorias de alguna forma aún no experimentada.

En caso de que las metodologías utilizadas sean diversas, entonces pueden establecerse argumentos para defender la aplicabilidad de una u otra para temas similares o diferentes. Si las opciones epistemológicas no concuerdan, entonces será necesario establecer las 
implicaciones sobre la metodología, la teoría y el hallazgo. Es recomendable siempre optar por una postura epistemológica congruente con la del investigador, donde se elimine siempre cualquier dogma de tipo filosófico. De esta manera, como lo refiere Sánchez Puentes (2004), la discusión bibliográfica permite elaborar un marco referencial de cómo se piensa enfrentar el tema de interés y facilitar la evaluación de opciones disponibles para abordar la investigación, las ventajas y desventajas de cada una y la razón del por qué de ellas. Aunque este marco referencial es muy parecido al tradicional, muchos investigadores tienden a interpretarlo como esencialmente teórico y obviarse los debates y opciones de tipo metodológico, empírico y hasta epistemológico. Por ello, el concepto de marco referencial obliga a incorporar en el esquema de trabajo los cuatro aspectos de la investigación ya referidos. Este es un componente esencial en la construcción del objeto de estudio porque, por un lado, ayuda a identificar exactamente lo que se quiere saber (basándose en lo que se sabe y no se sabe sobre el tema) y, por otro, cuál es la mejor forma de abordarlo (planteamientos teóricos, metodológicos y epistemológicos que mejor sirven). Es decir, como lo plantean Hernández y Barriga (2003:p.43), "el marco referencial permite delimitar el objeto a construir y da algunas ideas de cómo puede construirse. Esta delimitación habrá de ser siempre elaborada con rigurosidad y paciencia para ser realmente trascendente".

Esquema para la defini ción teórico-referencial de un objeto de estudio en Comunicación

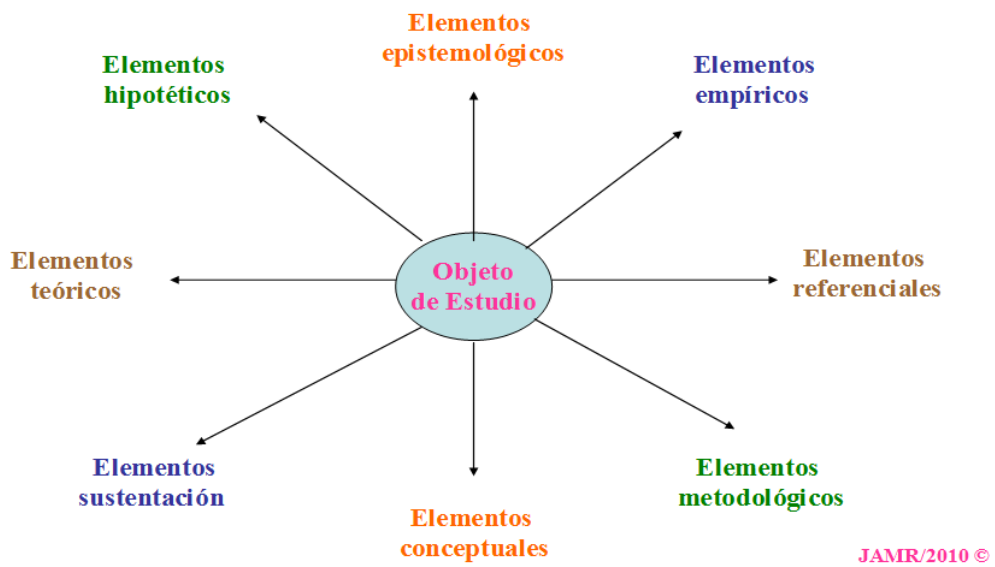

\subsection{Cuarta observación}

Una vez identificado el objeto a construir, la delimitación se convierte en el centro del proceso. El primer componente es la elaboración conceptual del objeto o, como precisa De 
Alba (1996.p.23), "la elaboración del objeto conceptual". El segundo es la estructuración del objeto empírico y el tercero la construcción final del objeto de estudio. Esta elaboración es la primera aproximación al cómo de la construcción del objeto y puede dividirse en tres grandes fases:

- Definición de un objetivo general y aquello que debe hacerse para lograr una adecuada construcción del objeto

- Vínculo entre el objeto (el conocimiento que se quiere construir) y el objetivo (la acción que debe realizarse para lograr esa construcción)

- Acción concreta de analizar las consecuencias sociales para lograr la construcción final del objeto.

En la reformulación del objeto es conveniente anteponer un verbo en infinitivo. Así, puede escribirse: "Analizar la relación entre el rendimiento escolar y los consumos intensivos de televisión de entretenimiento en los niños de educación primaria de las escuelas públicas de determinada ciudad". En esa misma orientación si el objetivo general es el destino, los objetivos específicos constituyen el itinerario. Es decir, indican las etapas parciales para cumplir con el objetivo general al final del proceso. En consecuencia, el objetivo general en las etapas intermedias puede ser:

- Identificar los casos a estudiar

- Identificar el rendimiento escolar de esos casos

- Identificar los niveles de consumo intensivo de televisión de entretenimiento en esos casos

- Establecer las relaciones entre el rendimiento escolar y los niveles de consumo intensivo de televisión de entretenimiento

- Examinar las relaciones para establecer causalidad.

Esta formulación de los objetivos específicos es, de acuerdo con Garza (2004), sumamente útil en tanto permite determinar tanto el diseño metodológico como el mapa de la ruta. Asimismo, porque define las actividades concretas a cumplir en cada etapa según los objetivos específicos. 


\subsection{Quinta observación}

La última fase para la elaboración del objeto conceptual reside en la elaboración de las hipótesis, sean estas formales o de trabajo. Por una serie de inercias formativas es común en el espacio iberoamericano desdeñar la noción de que toda investigación tiene un sustrato hipotético. Sin embargo, como lo señala Álvarez-Gayou (2005), "aunque es cierto que la hipótesis formal del modelo hipotético-deductivo no es un requisito sine qua non de la investigación científica, el analista como sujeto único es también un mito del relativismo científico". En ese sentido, es justo reconocer que el simple hecho de hacerse ciertas preguntas sobre un tema de interés es una evidencia concreta de que existen algunas ideas subyacentes de cómo opera el fenómeno, las nociones que orientan la búsqueda y los planteamientos inherentes. En esa dimensión, siempre existen de una u otra manera ciertas suposiciones u hipótesis. Por ello, al delimitar el objeto a construir y distribuir el objeto conceptual en objetivos es posible identificar el rumbo de la búsqueda, porque en este pueden encontrarse las ideas implícitas o explícitas que lo sustentan a partir de un marco referencial y otras reflexiones adicionales. De ellas pueden definirse las hipótesis de trabajo y, si es el caso, las hipótesis formales.

Al definirse el objeto conceptual, es posible realizar el trabajo de campo y construir el objeto empírico, es decir, hacer las observaciones y operacionalizarlas de forma tal que se logren los objetivos y, por extensión, la construcción del objeto de estudio. En la construcción del objeto empírico es posible también definir un diseño metodológico que determine cómo lograr los objetivos de forma concreta. Por tanto, es preciso incorporar los seis componentes básicos: 


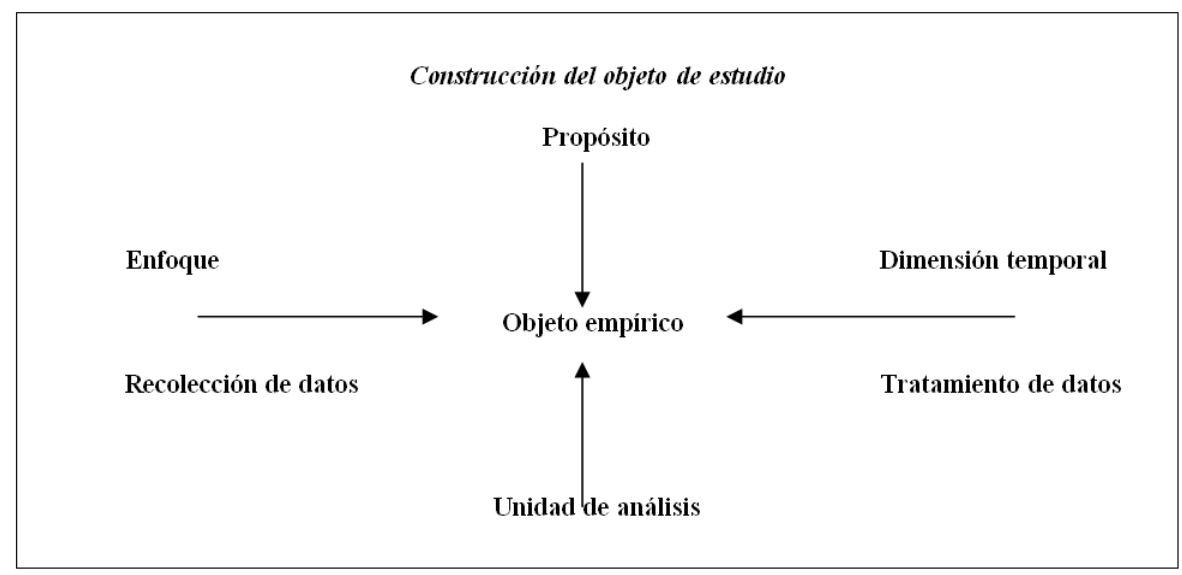

El diseño se nutre de estos elementos para poder definir claramente los procedimientos a realizar y el por qué de cada uno de ellos. El propósito, por su parte, identifica el para qué se realizan estas observaciones, ya sea para explicar, describir, explorar o evaluar. Este último se define con verbo en infinitivo, al igual que el objetivo general. Más aún, es permisible que el verbo utilizado en el objetivo general sea el mismo que el del propósito de investigación. Para el caso de los estudios en Comunicación ese rubro tiene tres grandes divisiones:

- Características del fenómeno. (Elemento estático del fenómeno de interés)

- Acciones vinculadas al fenómeno. (Aspectos dinámicos del fenómeno de interés)

- Orientaciones o motivaciones vinculadas al fenómeno. (Aspectos subjetivos sobre el fenómeno de interés)

Ninguna de estas tres opciones es superior a la otra, ni mutuamente excluyente. Incluso puede decirse que la Comunicación como área del conocimiento social requiere considerar los tres enfoques para desarrollar visiones completas de los fenómenos que le interesan. Esta dimensión temporal establece, como dice Garza (2004), cuándo se quieren hacer las observaciones 0 , incluso, distinguirse entre una investigación longitudinal y otra transversal. En el área de Comunicación la unidad de análisis busca saber lo qué se quiere observar y a quienes se quiere observar: individuos, grupos, organizaciones o segmentos sociales. Al definirse qué/quién quiere observar, es pertinente establecer también donde se quiere observar. Esta reflexión lleva a la noción del grupo social de interés, el cruce entre los casos y el contexto. De igual forma, en la discusión de la unidad de análisis, la definición de un 
grupo presenta la necesidad de definir cuáles de los posibles elementos de esa población van a ser observados.

En el contexto iberoamericano muchos investigadores consideran el proceso de muestreo como un procedimiento vinculado estrictamente a las metodologías cuantitativas, cuando en realidad distintos autores (Callejo, 2001) ya utilizan con frecuencia la palabra muestra para señalar la selección de casos en metodologías cualitativas. De cualquier forma, la extracción de una muestra es un proceso de selección de hechos a observar aunque este sea solamente uno. La recolección de datos es también la fase del diseño metodológico que señala el tipo de información que desea extraerse de esas observaciones. Este es uno de los aspectos más difíciles del proceso donde, como explica Rizo (2008), "la construcción de instrumentos transita del concepto abstracto al indicador concreto pasando por todas las subdimensiones posibles".

La aplicación del instrumento y la preparación de la base de datos entendida como la organización de la información recopilada, son los dos elementos constituyentes de esta etapa de recolección. En esta parte se busca conocer que operaciones quieren realizarse con esos datos observados y cómo piensa organizarse, resumirse o relacionarse la información recabada. El tratamiento son los procedimientos que se aplican a la información, mientras que el análisis se refiere al sentido que se otorga a esos procedimientos en la construcción del objeto. El último desafío consiste en cerrar el círculo analítico para, después de las elaboraciones conceptuales y empíricas, sea posible construir el objeto de estudio delimitado en el marco referencial. Es decir, como expresa Aguilar (2007) "el objetivo final de la investigación es lograr la representación de un fenómeno de interés y construir un objeto de estudio de una forma transparente y rigurosa que haga extensiva la forma en que y como se construyó".

\section{Consideración no final}

La forma de estructurar un objeto de estudio es fundamental para el alcance y significación de la investigación en Comunicación. Primero, porque permite determinar una estrategia de trabajo frente a una situación probable y, segundo, porque logra transparentar ante los interesados cómo se realizó la investigación. Con ello se provoca que los debates epistemológicos se aborden desde posturas mucho más relevantes. En ese sentido, el método de construcción propuesto se sustenta en la idea de que el objeto de estudio ha de 
definir la metodología más apropiada para la investigación y superar el debate tradicional entre investigaciones cualitativas y cuantitativas.

Adicionalmente, como lo ha señalado Galindo (2009), la discusión epistemológica que cuestiona la forma de trabajo de la Comunicación a partir de otras áreas del conocimiento social se asume desde una plataforma mejor sustentada y mayormente enfocada a la fundamentación que a la dispersión. Por lo anterior, resulta pertinente plantearse: ¿cómo desarrollar una epistemología desde la investigación en Comunicación (y no sobre la investigación social) que le otorgue un protagonismo central al objeto de estudio?. La respuesta por supuesto no es fácil y requiere de investigaciones constantes que lo validen. Sin embargo, su simple enunciación y reflexión periódicas plantean un escenario de análisis y reto fascinantes.\#

\section{Referencias}

- Aguilar, V. Notas para un debate sobre epistemología del discurso educativo, en Revista de Educación y Desarrollo No 7. México, Octubre-diciembre de 2007.

- Álvarez-Gayou, J. L. (2005). Cómo hacer una investigación cualitativa. Fundamentos y metodología. México: Editorial Paidós.

- Bourdieu, P., Chamboredon, J. C. y Passeron, J. C. La construcción del objeto, en Bourdieu, P. (1996). El oficio de sociólogo. Madrid: Siglo XXI.

- Callejo, J. (2001). El grupo de discusión: Introducción a una práctica de investigación. Barcelona: Editorial Ariel.

- De Alba, A. El objeto de estudio en la investigación. Diversas aproximaciones, en De Alba, A. (Coord.). (1996). Teoría y educación. Entorno al carácter científico de la educación. México: Universidad Nacional Autónoma de México.

- Díaz, A. La explicación científica. Una polémica desde la teoría del conocimiento, en Hoyos, C. (Coord.). (1997). Epistemología y objeto pedagógico ¿Es la pedagogía una ciencia?. México: Plaza y Valdés.

- Domínguez, S. (2007). El objeto de estudio en la investigación. Diversas aproximaciones. Revista Educación y Desarrollo No. 7. Guadalajara, octubrediciembre de 2007. 
- Galindo, J. Comunicología y sociedad de la información: Exploración de un lugar común desde la Ciencia de la Comunicación, en Revista Global Media Journal en español No. 11. Monterrey, otoño de 2009.

- Garza, A. (2004). Manual de Técnicas de Investigación para Estudiantes de Ciencias Sociales. México: El Colegio de México.

- Hernández, G y Barriga, O. La precisión del objeto de estudio, en Revista Cinta de Moebio No. 17. Santiago, septiembre de 2003.

- Hidalgo, J. L. Construcción del objeto de investigación. Una estrategia constructivista, en Revista de Investigación Educativa No. 16. México, agosto de 1992.

- Hoyos, C. (Coord.). (2005). Epistemología y objeto pedagógico ¿Es la pedagogía una ciencia?. México: Plaza y Valdés

- Karam, T. Epistemología y comunicación. Notas para un debate, en Revista Razón y Palabra No. 61. Atizapan, julio de 2008.

- Klimovsky, G. y de Asúa, M. (1997). Corrientes epistemológicas contemporáneas. Buenos Aires: Editores de América Latina.

- Lozano, J. C. (1996). Teoría e investigación de la comunicación de masas. México: Alhambra Mexicana.

- Navarro, R. (2006). Diseño de Proyectos de Investigación en Ciencias Sociales y Humanidades. México: Psicom Ediciones.

- Popper, K. (1973). La lógica de la investigación científica. Madrid: Tecnos

- Rizo, M. Ensayo general sobre la comunicación, en Revista Estudios sobre las Culturas Contemporáneas No. 48. Colima, octubre de 2008.

- Sánchez Puentes, R. (2004). Enseñar a investigar. Una didáctica nueva de la investigación en ciencias sociales y humanas. México: Plaza y Valdés y Universidad Nacional Autónoma de México.

- Sierra Bravo, R. (2007). Tesis doctorales y trabajos de investigación científica. Madrid: Editorial Paraninfo. 
José Antonio Meyer Rodríguez. Doctor en Ciencias de la Información por la Universidad de La Laguna (Tenerife, España). Autor de libros y artículos especializados sobre Comunicación política y desarrollo cultural. Miembro del Sistema Nacional de Investigadores Nivel 1 (Conacyt). Secretario de la Red Académica Iberoamericana de Comunicación y miembro activo de las asociaciones Latinoamericana de Investigadores de la Comunicación y Mexicana de Investigadores de la Comunicación. Evaluador del Consejo de Acreditación de la Comunicación y dictaminador de revistas especializadas sobre Comunicación en México y el exterior. Actualmente es Consejero Ciudadano de Transparencia en el Ayuntamiento de Puebla y coordinador del cuerpo académico Comunicación, Democracia y Desarrollo en la Universidad Popular Autónoma del Estado de Puebla (México).

\section{Forma de citar este artículo en bibliografías}

Meyer Rodriguez, J. A. (2010): "El objeto de estudio como sustento esencial de la investigación en Comunicación”, en Revista PANGEA, 1, páginas 108 a 123. Red Académica Iberoamericana de Comunicación. Recuperado el de de 2 de:

http://revistapangea.org/2010/12/05/01-01-106/ 